\title{
Nanocrystalline Cellulose Studied with a Conventional SEM
}

\author{
H. Sosiati ${ }^{1, *}$, M. Muhaimin ${ }^{2}$, Purwanto ${ }^{2}$, D.A. Wijayanti ${ }^{2}$ and K. Triyana ${ }^{1,2}$ \\ ${ }^{1}$ Nanomaterials Research Group, Integrated Research and Testing Laboratory (LPPT), Gadjah Mada University, Yogyakarta \\ 55281 , Indonesia. \\ ${ }^{2}$ Department of Physics, Faculty of Mathematics and Natural of Sciences, Gadjah Mada University, \\ Yogyakarta 55281, Indonesia. \\ Email: hsosiati@ugm.ac.id, hsosiati@gmail.com
}

\begin{abstract}
Nanocrystalline cellulose has been successfully studied by conventional scanning electron microscopy (SEM, JSM-6510LA). Nanocrystalline cellulose was extracted from the natural fibers by the chemical treatments of alkalization and bleaching followed by acid hydrolysis. Alkalization and bleaching resulted in microfibril cellulose, whereas a mix of micro and nanocrystallines cellulose resulted from acid hydrolysis after bleaching. Nanocrystalline cellulose separated from microcrystalline with a centrifuge at a rotation speed of $7000 \mathrm{rpm}$ and then dispersed in ethanol. Before SEM observation, nanocrystalline cellulose dispersed in ethanol was prepared on three different substrates; i.e. C-tape, Si-plate and C-coated $\mathrm{Cu}$ grid, and specimen surface was metallic coated. Crystallinity of the extracted cellulose was indicated from x-ray diffraction (XRD) analysis. SEM micrographs of nanocrystalline cellulose prepared on the C-coated grid showed the clearest morphologies. Crystalline cellulose nanowhiskers with length and width ranging between 300-600 nm and 40-60 nm, respectively, were clearly observed. SEM and transmission electron microscopy (TEM) images of the nanocrystalline cellulose were consistent. Dispersion time of nanocrystalline cellulose in ethanol was an important factor determining on the clarity of SEM/TEM micrograph. It is suggested that choosing a suitable sample preparation technique, conventional SEM is a powerful tool for the characterization of nanomaterials.
\end{abstract}

Keywords- Natural fiber, nanocellulose, SEM, XRD

\section{INTRODUCTION}

Studies on isolation of nanocrystalline cellulose from the natural fibers have recently attracted research interest around the world especially in the tropical countries where natural fibers are abundantly there. It is not only the availability of abundant natural fiber, but also natural fiber as a source of vegetable cellulose and its wide range applications. However, the properties of natural fiber are strongly dependence of age of fiber or harvest time, location of cultivation, climate and soil characteristic [1]. Accordingly, treatment conditions on the natural fibers obtained from different location to isolate micro and/or nanocellulose would be varied although their types/kinds are similar. Indonesian cultivated kenaf and sisal fibers must be hydrolyzed in relatively lower acid solution concentrations than that used by Kargarzadeh et al. [2] and Morán et al. [3]. Otherwise, they will shortly burn out [4]. This may be related to the fiber structure or the strength of fiber. Tensile strength of Indonesian raw kenaf is lower [5] than that of kenaf reported by Rouison et al. [6] cited in Akil et al. [7].

Fiber cells have of two layers of cell walls (Fig.1). The primary wall mostly contains disorderly networks of cellulose microfibrils. The secondary wall comprises three layers; i.e. S1, S2 and S3 containing helically arranged crystalline cellulose microfibrils. S2 is the thickest and this determines the strength of the fiber [1, 8]. By using chemical treatments to dissolve the amorphous phase, crystalline cellulose micro- and nano-fibrils can be isolated from the fiber structure.

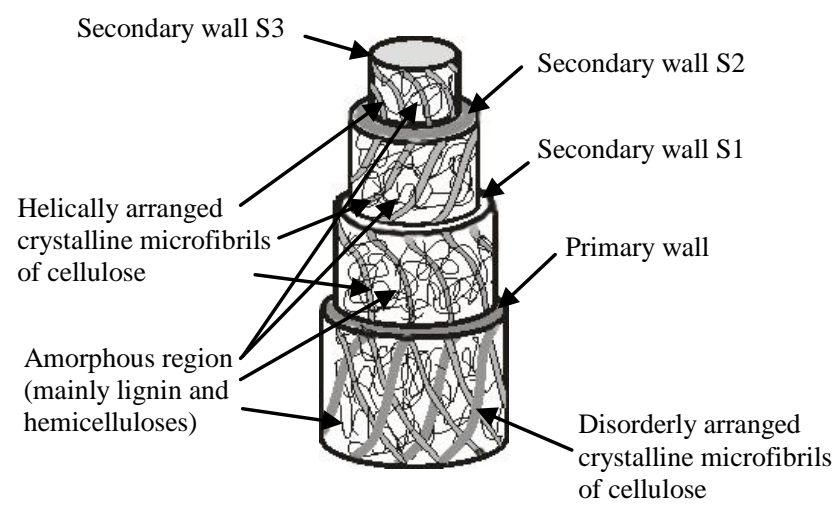

Figure 1. Schematic of the structure of fiber cell in a fiber plant [8]

The focus of this work is on a simple way to examine isolated nano-crystalline cellulose by conventional scanning electron microscopy (SEM). This can provide useful information for research scientists. SEM specimen preparation was conducted using tree kinds of substrate material: carbon tape (C-tape), silicon plate (Si-plate) and carbon coated $\mathrm{Cu}$-grid ( $\mathrm{C}$ coated $\mathrm{Cu}$-grid). The specimen preparation technique is a very important factor for getting qualified SEM and/or transmission electron microscopy (TEM) image. The morphologies of nano-crystalline 
cellulose resulting from these three different preparation methods were compared. The crystal phase of the extracted cellulose was indicated from $\mathrm{x}$-ray diffraction analysis.

\section{EXPERIMENTAL METHODS}

The natural fibers of kenaf (hibiscus cannabinus) and (sisal (agave sisalana) fibers obtained from Balittas, Malang, Indonesia. A route chemical treatment of alkalization in $\mathrm{NaOH}$, bleaching in a mix of $\mathrm{NaOH}$ and $\mathrm{H}_{2} \mathrm{O}_{2}$, and then acid hydrolysis in low concentration of $\mathrm{H}_{2} \mathrm{SO}_{4}$ under various conditions was subjected on dried kenaf and sisal to isolate the nanocrystalline cellulose. In this case, hydrolyzed cellulose contained a mix of micro- and nanocrystalline celluloses. Nanocrystalline cellulose was then separated from microcrystalline cellulose with a centrifuge at a rotation speed of $7000 \mathrm{rpm}$ for $5 \mathrm{~min}$ and repeated for about 3 steps until neutrality was achieved. Small amount of separated nanocrystalline cellulose was then put in ethanol and ultrasonically treated with (Power Sonic, LUC-405) to disperse nanocrystalline cellulose in ethanol homogeneously.

The morphology of nanocrystalline cellulose was observed using conventional SEM (JSM-6510LA) and TEM, (JEM-1400). X-ray diffraction (XRD, Bruker AXS-D8 Advance model system) was used to examine the crystallinity of the raw fiber and the hydrolyzed cellulose. The XRD was operated at $40 \mathrm{kV}$ and $40 \mathrm{~mA}$. XRD patterns were obtained from 2 theta; $10-50^{\circ}$ and at counting rate of $0.05 \%$.

Before SEM examination, nanocrystalline cellulose was prepared on the substrates $\mathrm{C}$-tape, $\mathrm{Si}$-plate and $\mathrm{C}$ coated $\mathrm{Cu}$ grid. A drop of nanocrystalline cellulose dispersed in ethanol was placed on each substrate. Prior to insertion into SEM specimen chamber each prepared, each specimen was dried and its surface coated with Pt (JEC-3000FC) to avoid the electrical charging.

\section{RESULTS AND DISCUSSION}

\section{A. SEM observations}

The surface morphology of the raw fiber and microfibrillated cellulose consisted of an amorphous phase of lignin and hemicelluloses covering the cellulose microfibrils (Fig. 2a; see arrows). The lignin and hemicelluloses dissolved in the chemical solutions during treatment, leading to fibrillation of the cellulose microfibers (Fig. 2b). The crystalline cellulose microfibril networks were arranged in a disorderly manner (see arrows) as schematically shown in Fig.1 [2].

Acid hydrolysis changed the crystalline microfibrillated cellulose to nanocrystalline cellulose. The morphologies of nanocrystalline cellulose were observed on three substrates. Characterization of nanocrystalline cellulose by SEM has been previously reported by some research groups [9-13], but they mainly used the advanced SEM or higher grade SEM than the conventional SEM used in this study. To date, no other studies have reported conventional SEM images of nanocrystalline cellulose.
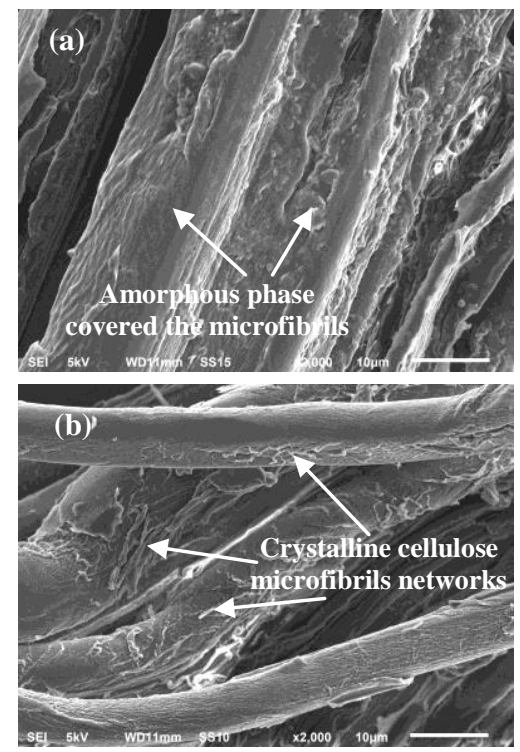

Figure 2. Surface morphology of kenaf raw fiber (a) and bleached fibers after alkalization (b).

Figure 3a demonstrates an SEM image of nanocrystalline cellulose prepared on a C-tape substrate showing the morphology of nanosphericals and nanorods (see arrows).
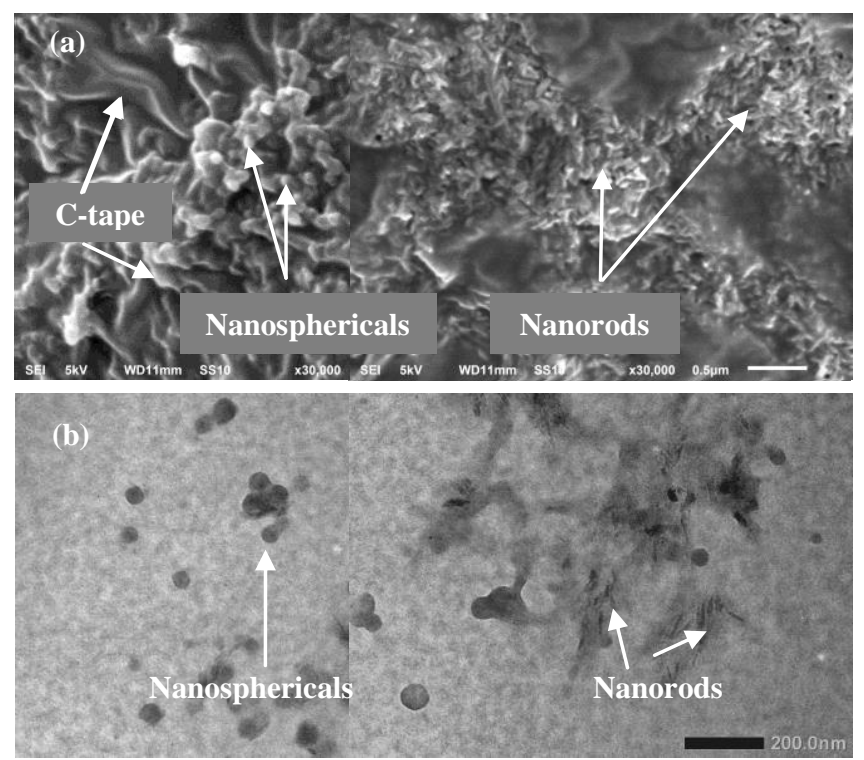

Figure 3. SEM image of nanocrystalline cellulose prepared on a C-tape which dried imperfectly (a) and TEM image of the same specimen (b).

After a small amount of nanocrystalline cellulose dispersed in ethanol was dropped onto the substrates, it should be left in air for about $15 \mathrm{~min}$ and then dried using a hair drier until perfectly dried. Imperfectly drying led to indistinct morphologies of both nanosphericals and nanorods (Fig. 3a), 
because the strong bombardment of electron beam hitting the carbon tape surface made it wrinkled (see arrows). This image is slightly clearer in TEM (Fig. 3b). Cellulose nanosphericals with average diameter around $50 \mathrm{~nm}$ and cellulose nanorods of 10-15 $\mathrm{nm}$ in width and $\sim 100 \mathrm{~nm}$ in length were observed. However, the nanorods were still slightly unclear because they remained distributed within the residual amorphous phase. Single application of alkalization and bleaching resulted in two types of morphology (Fig. 3). If done several times (more than twice), only cellulose nanowhiskers were produced. Repeated alkalization and bleaching led to removal of most of the amorphous phase and increased the degree of fiber fibrillation.

SEM images of crystalline cellulose nanowhiskers prepared on a C-tape and a Si-plate which dried perfectly are displayed in Fig. $4 \mathrm{a}$ and $4 \mathrm{~b}$, respectively. These morphologies of the nanowhiskers are clearer than those in Fig. 3a, indicating that specimen preparation technique is significant for getting a good image. The SEM image in Fig. 4(a) appears clearer than that in Fig. 4(b). This might be caused by the differences between the substrate surface. However, if the density of the nanowhiskers on the substrate surface was too high, indistinct morphologies of individual nanowhisker result.
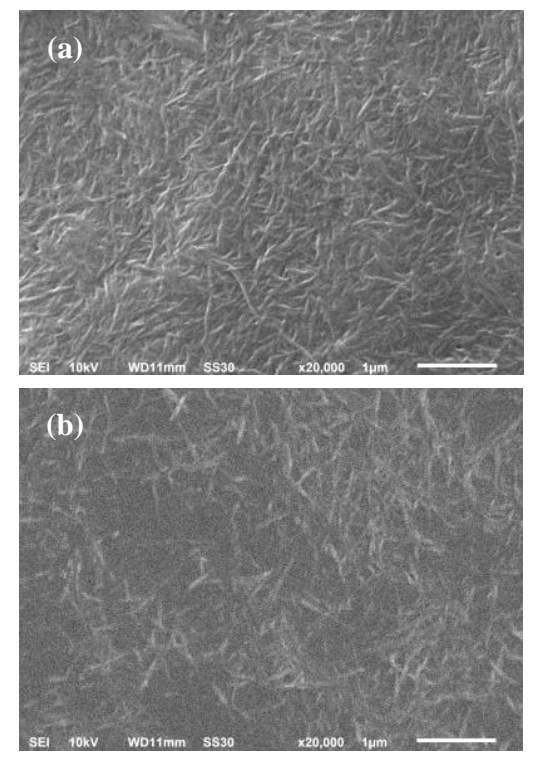

Figure 4. SEM images of nanocrystalline cellulose prepared on perfectly dry of a C-tape (a) and a Si plate (b) substrates.

Fig. 5 exhibits crystalline cellulose nanowhiskers prepared on a $\mathrm{C}$ coated Cu-grid. The dispersal time of the nanocrystalline cellulose in ethanol before preparing the substrate is another important factor affecting the quality of the SEM image of nanocrystalline cellulose. Fig. 5a shows an SEM image of nanocrystalline cellulose with a dispersion time of $10 \mathrm{~min}$. The nanofibers appear not to be separated from each other leading to a nebulous morphology. Prolonging the dispersion time to $30 \mathrm{~min}$ gave a much better image (Fig. 5b). Crystalline cellulose nanowhiskers with length and width ranging between 300-600 nm and 40-60 $\mathrm{nm}$, respectively, were clearly observed. Bright contrast of fine particles distributed across the entire area is interpreted as a residual effect of the hydrolysis solution due to its incomplete neutralization. This should be avoided. The quality of the morphology of crystalline cellulose nanowhiskers in Fig. 5b is the clearest and comparable with that observed with atomic force microscope (AFM) [3], advanced SEM (FE-SEM, ZEISS-ULTRA55) [6] (FE-SEM, FEI Quanta 200F) [7] and SEM (Hitachi-4700) [8]. This suggests that by good choice of specimen preparation technique, conventional SEM can be a powerful tool for characterization of nanomaterials. This study has also shown that crystalline cellulose nanowhiskers can be successfully isolated from natural fibers with high repeatability.
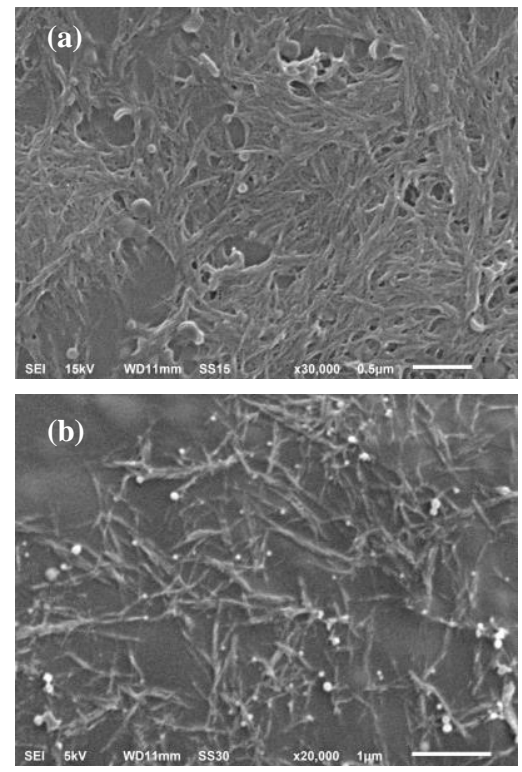

Figure 5. SEM images of nanocrystalline cellulose prepared on a carbon coated $\mathrm{Cu}$-grid with different dispersion time; (a) $10 \mathrm{~min}$ and (b) $30 \mathrm{~min}$.

\section{B. XRD analysis}

XRD profiles of raw fiber and hydrolyzed cellulose are depicted in Fig. 6 show different patterns. Peaks of raw fiber are located at 2 theta around $16.10^{\circ}\left(I_{-111}\right.$ lattice reflection), $22.30^{\circ} \quad\left(I_{002}\right.$ lattice reflection) and $34.60^{\circ} \quad\left(I_{-231}\right.$ lattice reflection), whereas those at of hydrolyzed cellulose area placed at 2 theta $14.90^{\circ}\left(I_{011}\right.$ lattice reflection $), 16.49^{\circ}\left(I_{-111}\right.$ lattice reflection), $22.64^{\circ}\left(I_{002}\right.$ lattice reflection $), 29.06^{\circ}\left(I_{-122}\right.$ lattice reflection) and $34.06^{\circ}\left(I_{310}\right.$ lattice reflection) (native cellulose, PDF \# 030289). The peaks in the XRD profile of hydrolyzed cellulose are sharper than those of raw fiber, indicating that the volume fraction of the crystalline phase contained within the hydrolyzed cellulose is higher compared with that in raw fiber. However, a low intensity broad peak located at 2 theta $29.06^{\circ}$ was identified. This showed that most of the amorphous phase contained in raw fiber dissolves in the chemical solution after hydrolysis. In 
other words, acid hydrolysis increased the crystallinity and produced nanocrystalline cellulose or cellulose nanocrystal.

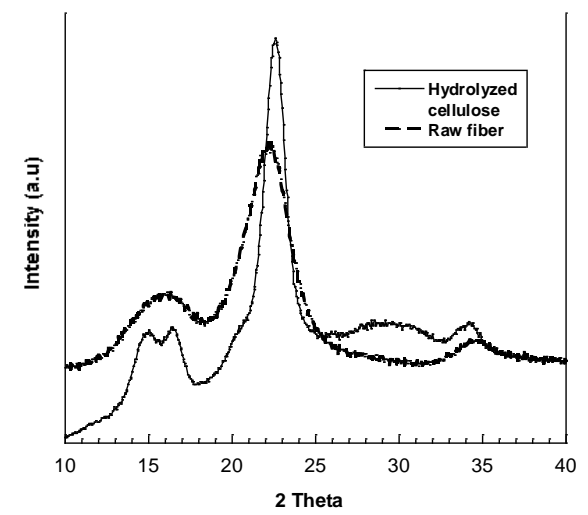

Figure 6. XRD profiles of the raw fiber (a) and hydrolyzed cellulose microfibrils (b).

\section{CONCLUSIONS}

Nanocrystalline cellulose has been successfully characterized by means of a conventional SEM (JSM6510LA).

1. Crystalline cellulose nanowhiskers with length and width ranging between $300-600 \mathrm{~nm}$ and 40-60 nm, respectively, were clearly observed following good specimen preparation technique and using a $\mathrm{C}$ coated $\mathrm{Cu}$-grid.

2. C coated $\mathrm{Cu}$-grid is a better substrate material for preparing SEM specimens of nanocrystalline cellulose or other nanomaterials than C-tape and Si-plate. It results in a clearer morphology of the specimen.

3. Optimum dispersion time of nanocrystalline cellulose in ethanol before specimen preparation on the substrate is around $30 \mathrm{~min}$.

4. Repeatability of isolating crystalline cellulose nanowhiskers can be achieved.

\section{ACKNOWLEDGMENT}

The authors would like to thank the "RISET UNGGULAN PERGURUAN TINGGI", Gadjah Mada University for funding this project (LPPMUGM/1228/LIT/2013 and LPPM-UGM/547/LIT/2014). Deep appreciation also goes to Mr. Kunto Wandono for his assistant to design a high speed mixer.

\section{REFERENCES}

[1] S. Kaila, A. Dufresne, B.M. Cherian, B.S. Kaith, L. Averous, J. Njuguna and E. Nassiopoulos, "Cellulose-based bio-and nanocomposites: A. review", International Journal of Polymer Science. Vol. 2011, pp. 1-35, 2011.
[2] H. Kargarzadeh, I. Ahmad, I. Abdullah, A Dufresne, S.Y. Zainudin and R.M. Sheltami, "Effect of hydrolysis conditions on themorphology, crystallinity, and thermal stability of cellulose nanocrystals extracted from kenaf bast fibers", Cellulose. Vol. 19, pp. 855-866, March 2012.

[3] J.I. Morán, V.A. Alvarez, V.P.Cyras and A. Vázquez, "Extraction of cellulose and preparation of nanocellulose from sisal fibers", Cellulose, vol. 15, pp. 149-159, 2008

[4] Purwanto, "Characterization of isolated micro- and nano-cellulose from kenaf and sisal fibers", Master Thesis, 2014

[5] H. Sosiati, Ar Rohim, Ma'arif, K. Triyana and Harsojo, " Relationships between tensile strength, morphology and crystallinity of treated kenaf bast fibers", AIP Conf. Proc., vol. 1554, pp. 42-46, 2013.

[6] D. Rouison, M. Sain and M. Couturier, " Resin tranfer molding of natural fiber reinforced composites: cure simulation", Comp. Sci. Technol., vol.64, pp.629-644, 2004.

[7] H.M. Akil, M.F. Omar, A.A.M. Mazuki, S. Safiee, Z.A.M. Ishak and A. Abu Bakar, "Kenaf fiber reinforced composites: A review", Materials and Design, vol. 32, pp. 4107-4121, 2011.

[8] M.Z. Rong, M.Q. Zhang, Y. Liu, G.C. Yang and H.M. Zeng, "The effect of fiber treatment of the mechanical properties of undirectional sisal-reinforced epoxy composites", Composite Sci. Technol., vol. 61, pp. 1437-1447, 2001.

[9] M.M. Kabir, H. Wang, K.T. Lau and F. Cardona, "Chemical treatment on plant-based natural fiber reinforced polymer composite: An overview", Composites Part B, vol. 43 (7), pp. 2883-2892, May 2012

[10] M. Roohani, Y. Habibi, N.M. Belgacem, G. Ebrahim, A.N. Karimi and A. Dufresne, "Cellulose whiskers reinforced polyvinyl alcohol copolymers nanocomposites", European Polymer Journal, vol. 44, pp. 2489-2498, June 2008.

[11] A. Kumar, Y.S. Negi, V. Choudhary and N.K. Bhardwaj, "Characterization of cellulose nanocrystals produced by acidhydrolysis from sugarcane bagasse as agro waste", J. Mater. Phys. Chem., vol. 2(1), pp. 1-8, 2014.

[12] M. Ioelovich, "Study of cellulose interaction with concentrated solutions of sulfuric acid", ISRN Chemical Engineering, vol. 2012, pp. 1-7, 2012.

[13] D. Gaspar, S.N. Fernandes, A.G. de Oliveira, J.G. Fernandes, P. Grey, R.V. Pontes, L. Pereira, R. Martins, M.H. Godinho and E. Fortunato, "Nanocrystalline cellulose applied simultaneously as the gate dielectric and the substrate in flexible field effect transistors", Nanotechnology, vol. 25 (9), pp. 1-11, February 2014. 